\title{
Image of the month: Dysphagia due to injury of the corticobulbar tract following traumatic brain injury
}

Authors: Sung Ho Jang, ${ }^{A}$ Seong Ho Kim ${ }^{B}$ and Jeong Pyo Seo ${ }^{C}$

A 51-year-old male patient was diagnosed with traumatic epidural hematoma in the left frontal area and contusional hematoma in both prefrontal areas resulting from a fall after being hit by a truck while walking in a side street, in which the patient's head hit the ground. The patient lost consciousness for approximately 45 days and experienced continuous post-traumatic amnesia from the time of the accident. His Glasgow Coma Scale score was 8 when he arrived at the hospital. He underwent conservative management at the department of neurosurgery of a local hospital. After recovering consciousness, he felt dysphagia for water or watery food. When he drank water, he usually showed aspiration such as severe coughing. The conventional brain MRI taken at approximately four months did not show abnormality along the pathway of the corticobulbar tract (CBT) (Fig 1a). ${ }^{1}$ Six months after the trauma, he visited the
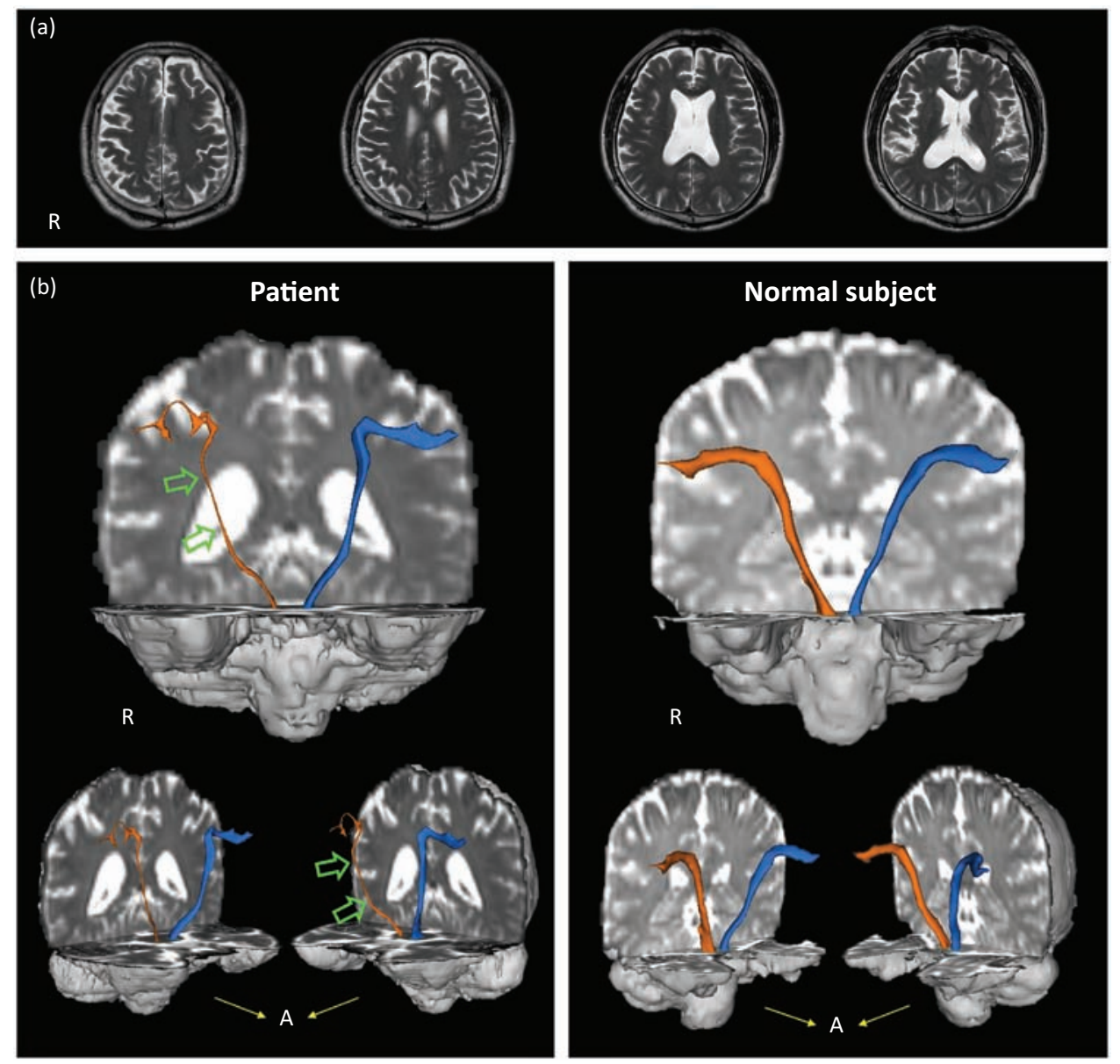

Fig 1. The MR images and result of diffusion tensor tractography. (a) T2-weighted brain MR images taken at four months do not show abnormality along the pathway of the corticobulbar tract (CBT). (b) Results of diffusion tensor tractography for the CBT. The right CBT revealed severe narrowing (arrows) compared with the left CBT and those of a normal subject (44-year old female).

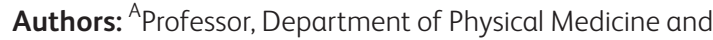
Rehabilitation, College of Medicine, Yeungnam University; ${ }^{B}$ Professor, Department of Neurosurgery, College of Medicine Yeungnam University; ${ }^{\mathrm{C}} \mathrm{PhD}$, Department of Physical Medicine and Rehabilitation, College of Medicine, Yeungnam University rehabilitation department of a university hospital for evaluation of dysphagia.

Diffusion tensor imaging (DTT) data were acquired at five months after onset using a 6-channel head coil on a 3 T Philips Gyroscan Intera (Philips, Ltd, Best, The Netherlands) with single-shot echo-planar imaging. Fibre tracking was performed using the Oxford Centre for Functional Magnetic Resonance 
Imaging of the Brain (FMRIB) Software Library (FSL; www. fmrib.ox.ac.uk/fsl). The seed region of interest (ROI) was placed at the lower portion of the precentral gyrus on the axial slice in which the top of the lateral ventricle could be seen, and the target ROI was placed on the lower pons of the anterior blue portion on the color map. ${ }^{2}$ The right CBT showed severe narrowing compared with the left CBT (Fig 1b).

In this case study, we found narrowing of the right CBT on DTT in a patient with dysphagia following traumatic brain injury. Severe narrowing of the right CBT on DTT indicates neural injury and this injury of the right CBT likely contributed to the dysphagia in this patient. Because no definite brain lesion was detected on conventional brain MRI, traumatic axonal injury was the most likely pathogenetic mechanism. ${ }^{3,4}$ We think that DTT for the CBT would be helpful for detecting the lesion for patients with swallowing difficulty following traumatic brain injury.

\section{Acknowledgements}

This work was supported by the DGIST R\&D Program of the Ministry of Science, ICT and Future Planning (17-BD-0401).

\section{References}

1 Jang SH, Seo JP. The anatomical location of the corticobulbar tract at the corona radiata in the human brain: Diffusion tensor tractography study. Neurosci Lett 2015;590:80-3.

2 Jang SH, Lee J, Kwon HG. Reorganization of the corticobublar tract in a patient with bilateral middle cerebral artery territory infarct. Am J Phys Med Rehabil 2016;95:e58-9.

3 Povlishock JT, Christman CW. The pathobiology of traumatically induced axonal injury in animals and humans: A review of current thoughts. J Neurotrauma 1995;12:555-564.

4 Buki A, Povlishock JT. All roads lead to disconnection? - traumatic axonal injury revisited. Acta Neurochir (Wien) 2006;148:181-93.

Address for correspondence: Department of Physical Medicine and Rehabilitation, College of Medicine, Yeungnam University 317-1, Daemyungdong, Namku, Taegu, 705-717, Republic of Korea.

Email: raphael0905@hanmail.net

\section{Royal College of Physicians}
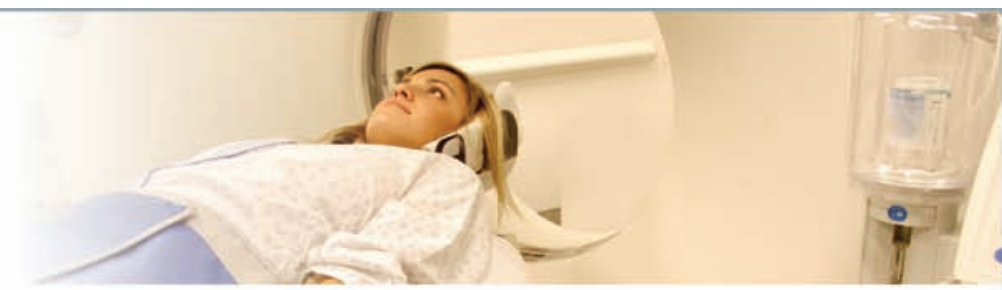
Medical Care

\section{Are you involved in planning nuclear medicine services?}

\begin{abstract}
Medical Care is the online evolution of the well-known RCP publication Consultant physicians working with patients. Written by leading medical specialty experts, it is a comprehensive web-based resource for the efficient and effective design of medical services covering all the specialties including nuclear medicine.
\end{abstract}

www.rcpmedicalcare.org.uk
The resource looks in detail at the services delivered by nuclear medicine, including:

> standard diagnostic imaging

$>$ nuclear cardiology

$>$ non-imaging tests

$>$ sentinel node services

$>$ PET services

$>$ therapeutic nuclear medicine

$>$ neuroendocrine tumour services

> bone-targeting molecular radiotherapies.

Medical Care has been designed to help those involved in the planning and provision of medical services to get a clearer picture of the specialty services that need to be in place to provide great patient care. 